\title{
Erratum: Field-effect induced tunability in hyperbolic metamaterials [Phys. Rev. B 92, 184101 (2015)]
}

\author{
Georgia T. Papadakis and Harry A. Atwater \\ (Received 16 November 2017; published 27 November 2017)
}

DOI: 10.1103/PhysRevB.96.179903

In the original paper, we illustrated the potential for tunable birefringence and dichroism in metamaterial heterostructures where a field-effect gated structure is used to change the carrier density and modulate the permittivity in transparent conductive oxides. A layered heterostructure is proposed for which active modulation leads to changes in the effective electromagnetic parameters, enabling active control of the birefringence, dichroism, and anisotropic dispersion of hyperbolic metamaterials. Following publication, we found errors in the values for the carrier density and breakdown field reported in Table I of the paper. Our revisions to Table I correct typographical errors and reflect more accurate evaluations of the reported breakdown fields achievable in hafnium oxide $\left(\mathrm{HfO}_{2}\right)$. These corrections reduce the estimated size of the achievable effects somewhat but do not alter the conceptual framework described in the paper or the predicted behavior for metamaterial parameters at a given carrier density value.

TABLE I. Maximum achievable carrier concentration in indium tin oxide (ITO) accumulation layers for reported values of the breakdown field and de dielectric constants of high-strength dielectrics.

\begin{tabular}{|c|c|c|c|c|c|}
\hline Dielectric & $E_{\mathrm{b}}$ and $k_{\text {diel }}$ & $\begin{array}{c}N\left(\text { in } 10^{21} \mathrm{~cm}^{-3}\right) \\
d=0.5 \mathrm{~nm}\end{array}$ & $\begin{array}{c}N\left(\text { in } 10^{21} \mathrm{~cm}^{-3}\right) \\
d=1 \mathrm{~nm}\end{array}$ & $\begin{array}{c}N\left(\text { in } 10^{21} \mathrm{~cm}^{-3}\right) \\
\quad d=2.5 \mathrm{~nm}\end{array}$ & $\begin{array}{c}N\left(\text { in } 10^{21} \mathrm{~cm}^{-3}\right), \\
d=5 \mathrm{~nm}\end{array}$ \\
\hline $\mathrm{SiO}_{2}$ & $\begin{array}{c}E_{\mathrm{b}}=[30-40] \mathrm{MV} / \mathrm{cm}[44] \\
k_{\mathrm{diel}}=3.9[44-46]\end{array}$ & [1.29-1.7] & [0.65-0.86] & [0.26-0.35] & [0.13-0.17] \\
\hline $\mathrm{Al}_{2} \mathrm{O}_{3}$ & $\begin{array}{c}E_{\mathrm{b}}=[6-8] \mathrm{MV} / \mathrm{cm}[45,47,48] \\
k_{\text {diel }}=9[45,46]\end{array}$ & {$[0.6-0.8]$} & {$[0.3-0.4]$} & {$[0.12-0.16]$} & [0.06-0.08] \\
\hline $\mathrm{Al}_{2} \mathrm{O}_{3}$ & $\begin{array}{c}E_{\mathrm{b}}=[6-8] \mathrm{MV} / \mathrm{cm}[45,47,48] \\
k_{\text {diel }}=10.3[48]\end{array}$ & [0.68-0.91] & [0.34-0.46] & {$[0.14-0.18]$} & [0.07-0.09] \\
\hline $\mathrm{HfO}_{2}$ & $\begin{array}{c}E_{\mathrm{b}}=[13] \mathrm{MV} / \mathrm{cm}[44] \\
k_{\text {diel }}=17[44]\end{array}$ & [2.4] & [1.2] & {$[0.48]$} & {$[0.24]$} \\
\hline $\mathrm{HfO}_{2}$ & $\begin{array}{c}E_{\mathrm{b}}=[13] \mathrm{MV} / \mathrm{cm}[44] \\
k_{\mathrm{diel}}=25[46]\end{array}$ & [3.6] & [1.88] & {$[0.72]$} & {$[0.36]$} \\
\hline $\mathrm{HfO}_{2}$ & $\begin{array}{c}E_{\mathrm{b}}=5.6 \mathrm{MV} / \mathrm{cm}[48] \\
k_{\text {diel }}=18.7[48]\end{array}$ & 1.16 & 0.58 & 0.23 & 0.12 \\
\hline $\mathrm{HfSiO}_{4}$ & $\begin{array}{c}E_{\mathrm{b}}=10 \mathrm{MV} / \mathrm{cm}[46,49] \\
k_{\text {diel }}=11[46,49]\end{array}$ & 1.22 & 0.61 & 0.24 & 0.12 \\
\hline $\begin{array}{l}\mathrm{HfSiO}_{4} \\
\text { (with } \mathrm{SiO}_{2} \text { ) }\end{array}$ & $\begin{array}{c}E_{\mathrm{b}}=10 \mathrm{MV} / \mathrm{cm}[46,49] \\
k_{\text {diel }}=[4.8-5.4][50]\end{array}$ & [0.53-0.6] & {$[0.27-0.3]$} & [0.11-0.12] & {$[0.05-0.06]$} \\
\hline $\begin{array}{l}\mathrm{HfSiO}_{4} \\
\text { (with } \mathrm{HfO}_{2} \text { ) }\end{array}$ & $\begin{array}{c}E_{\mathrm{b}}=10 \mathrm{MV} / \mathrm{cm}[46,49] \\
k_{\text {diel }}=[12.5-15.1][50]\end{array}$ & [1.38-1.67] & [0.69-0.83] & [0.28-0.33] & [0.14-0.17] \\
\hline
\end{tabular}

[44] C. Sire, S. Blonkowski, M. J. Gordon, and T. Baron, Appl. Phys. Lett. 91, 242905 (2007).

[45] C. M. Tanner, Y.-C. Perng, C. Frewin, S. E. Saddow, and J. P. Chang, Appl. Phys. Lett. 91, 203510 (2007).

[46] A. P. Huang, Z. C. Yang, and Paul K. Chu, Advances in Solid State Circuit Technologies, edited by P. K. Chu (InTech, Rijec, Croatia, 2010), Chap. 16.
[47] M. D. Groner, J. W. Elam, F. H. Fabreguette, and S. M. George, Thin Solid Films 413, 186 (2002).

[48] J. Yota, H. Shen, and R. Ramanathan, J. Vac. Sci. Technol., A b31, $01 \mathrm{~A} 134$ (2013).

[49] J. Robertson, Eur. Phys. J. Appl. Phys. 28, 265 (2004).

[50] Z. M. Rittersma, F. Roozeboom, M. A. Verheijen, J. G. M. van Berkum, T. Dao, J. H. M. Snijders, E. Vainonen-Ahlgren, E. Tois, M. Tuominen, and S. Haukka, J. Electrochem. Soc. 151, C716 (2004). 